\title{
Characterization of novel Actinobacteriophage Giantsbane reveals potential genomic recombination hotspot and unexpected intra-cluster similarities
}

Christopher Liu*, Pei Ying Chen*, Preston Dang ${ }^{*}$, Michael Zhang*, Andrew Kapinos, Ryan Ngo, Jordan Moberg Parker ${ }^{1}$, Amanda C. Freise ${ }^{2}$

*Denotes equal contributions.

Department of Microbiology, Immunology, and Molecular Genetics, University of California, Los Angeles (UCLA), Los Angeles, CA, USA

Contact Information

1jmobergparker@ucla.edu

${ }^{2}$ afreise@ucla.edu 


\title{
Characterization of phage Giantsbane
}

\begin{abstract}
Bacteriophages that infect Arthrobacter, a genus of bacteria which play key ecological roles in soil, warrant further study. A novel Actinobacteriophage, Giantsbane, was isolated on Arthrobacter globiformis and purified. Particle stability was tested at various temperatures and salinity concentrations by observing titer differences; unpaired Student's t-tests revealed these differences to be insignificant. Transmission electron microscopy and Illumina whole-genome sequencing revealed that Giantsbane's morphology and genome length, respectively, were characteristic of Siphoviridae phages. 94 putative open reading frames were determined using Glimmer, GeneMark, and manual review; none were associated with lysogeny. Giantsbane was placed into phage cluster AU, and SplitsTree and batch ANI analyses revealed similarities with other AU phages. The annotated genome was further analyzed using Phamerator and MEME-Suite, which identified repeated motifs present in several other phages. These findings help further our understanding of the physiological and genomic aspects of phage biology.
\end{abstract}

\section{INTRODUCTION}

Despite being the most abundant biological entities, bacteriophages are not well understood or characterized (Hatfull, 2015). Soil bacteriophages are of particular interest because of their ability to infect bacteria critical to ecological processes such as nutrient cycling (Uroz et al., 2011). Functionally and genetically characterizing these bacteriophages increases our understanding of their biological and ecological roles, and contributes to a significant and growing body of knowledge. Arthrobacter, a genus of soil bacteria, is a keystone taxon in soil ecosystems due to its ability to recycle nitrogen and carbon (Romaniuk et al., 2018). Its diverse 
Characterization of phage Giantsbane

metabolic capabilities affect the composition of nutrients in soil, impacting soil ecosystem biodiversity and health (Eschbach et al., 2003).

Phages shape the microbiomes of different environments (Zhang et al., 2017) and the ability of a phage to infect Arthrobacter depends on how well it survives in common soil environments (Iriarte et al., 2007). Determining how soil bacteriophages infect hosts under various environmental conditions reveals the boundaries within which the phage can propagate. Here we report the isolation and characterization of a novel phage, Giantsbane, isolated on host Arthrobacter globiformis.

To test the conditions under which Giantsbane might infect $A$. globiformis populations in soil, we examined how titer and plaque morphology changed as a result of temperature and salinity variations. Transmission electron microscopy (TEM) was used for morphological characterization. Illumina genome sequencing, genome analysis, and annotation enabled genomic characterization, and comparative genomic analysis determined Giantsbane's relation to other Arthrobacter-infecting phages.

Environmental condition assays showed that Giantsbane was resistant to temperature and salinity variations within $20-30^{\circ} \mathrm{C}$ and up to $0.12 \mathrm{M} \mathrm{NaCl}$, respectively. TEM revealed that Giantsbane was a member of the Siphoviridae family. Whole-genome sequencing and annotation revealed a genome of $56 \mathrm{kbp}$ and 94 putative genes, 27\% of which have putative functions.

Comparative analysis showed that Giantsbane shares high nucleotide sequence similarity with phages in cluster AU, which is composed of lytic, Siphoviridae phages. Genomic analyses revealed that cluster AU separates into four distinct groupings, based on nucleotide and pham similarities. A 32 bp repeat sequence found in Giantsbane and other phages may provide a basis 
Characterization of phage Giantsbane

for genetic mosaicism through homologous recombination as indicated by several gene translocations that only occur near the repeat sequences, increasing phage diversity.

\section{EXPERIMENTAL PROCEDURES}

\section{Sample Collection, Direct Isolation, and Spot Test}

Soil was collected on April 8th, 2019 at 2:40pm from the UCLA Botanical Gardens in Los Angeles, $\mathrm{CA}\left(34.065562^{\circ} \mathrm{N}, 118.441146^{\circ} \mathrm{W}\right)$, at $26.1^{\circ} \mathrm{C}$ from dry soil at a depth of $23.9 \mathrm{~cm}$. Sample soil was incubated with enrichment broth at $250 \mathrm{rpm}$ with shaking for 1.5 hours at $25^{\circ} \mathrm{C}$ and filtered through a $0.22 \mu \mathrm{m}$ syringe filter. Filtrate and $A$. globiformis strain B-2979 were mixed in a $2: 1$ ratio and incubated at $25^{\circ} \mathrm{C}$ for 20 minutes before being mixed with $2 \mathrm{X}$ PYCa soft agar, plated on a PYCa agar plate, and incubated at $25^{\circ} \mathrm{C}$ for 24 hours.

Putative plaque clearings were picked and diluted with phage buffer, and was added to a soft agar overlay of PYCa and A. globiformis, and incubated for $24-48$ hours at $25^{\circ} \mathrm{C}$ for a spot test to confirm phage presence.

\section{Plaque Assay Purification}

A clearing from the spot tests was picked and diluted in phage buffer. To purify the phage, two consecutive plaque assays were performed. Serial dilutions of phage stock from $10^{-1}$ to $10^{-9}$ were mixed with $A$. globiformis B-2979 at a $1: 10$ ratio, then incubated at $25^{\circ} \mathrm{C}$ for 10 minutes. This mixture was plated using a soft agar overlay with PYCa top agar and PYCa plates. Dried plates were incubated for 48 hours at $25^{\circ} \mathrm{C}$. 


\section{Characterization of phage Giantsbane}

\section{MINI and MIDI Lysate Collection and Titer Determination}

Purified plates exhibiting web lysis were flooded with phage buffer and incubated at $25^{\circ} \mathrm{C}$ for one hour. Contents were filter sterilized with a $0.22 \mu \mathrm{m}$ filter to create the MINI lysate. Lysate titer was determined through the standard plaque assay protocol, and used to calculate $\mathrm{PFU}_{\mathrm{MAX} \text { WEB }}$ for webbed-lysis on the MINI assay plates.

Plates showing web-lysis patterns from the MINI lysate plaque assay were subsequently flooded with phage buffer and incubated at $25^{\circ} \mathrm{C}$ for one hour before its contents were filter sterilized to create the MIDI lysate. Titer was calculated from a standard plaque assay protocol.

\section{Transmission Electron Microscopy}

Purified phage lysate was placed on a carbon-coated EM grid and left for 2 minutes.

Excess fluid was removed and $3 \mu \mathrm{L}$ of $1 \%$ uranyl acetate stain solution was added and left for 2 minutes. Staining was repeated twice. The grid was air-dried for 10 minutes and visualized with a Philips CM120 microscope. Measurements of capsids and tails were calculated with ImageJ.

\section{Temperature and Salinity Assays}

For each assay, plaque assays were performed under different conditions. Negative control plates contained only phage buffer and were subjected to the tested environmental conditions. Temperature assay plates were incubated at $20^{\circ} \mathrm{C}, 25^{\circ} \mathrm{C}$ (positive control), $30^{\circ} \mathrm{C}$, $33^{\circ} \mathrm{C}$, or $35^{\circ} \mathrm{C}$ for 48 hours. For the salinity assay, A. globiformis was mixed with aqueous $\mathrm{NaCl}$ to create $0 \mathrm{M}$ (positive control), $0.03 \mathrm{M}, 0.06 \mathrm{M}, 0.09 \mathrm{M}$, and $0.12 \mathrm{M} \mathrm{NaCl}$ solutions and incubated at $25^{\circ} \mathrm{C}$ for 10 minutes pre-adsorption. For all assays, plaque size, morphology and count were recorded and titers were calculated. Differences in titer for both assays were tested 
Characterization of phage Giantsbane

for significance with a one-tailed, unpaired Student's t-test at $\alpha=0.05$. Statistics were calculated and graphs prepared using Prism version 8 (Graphpad Software, CA, USA).

\section{DNA Extraction, Sequencing and Assembly Methodology}

Phage DNA was extracted using the Wizard DNA Clean-Up kit (cat \# A7280, Promega, WI, USA). Sequencing libraries were made using a NEB Ultra II Library Prep kit (New England Biolabs, MA, USA) and were sequenced using the Illumina MiSeq platform. Contigs were assembled using Newbler version 2.9 which was checked for accuracy and genomic termini using Consed version 29.

\section{Gene Location}

DNA Master version 5.0.2 (http://cobamide2.bio.pitt.edu/computer.htm) and PECAAN (https://pecaan.kbrinsgd.org/) were used for genome auto-annotation. Glimmer version 3.02 (Delcher et al., 2007) and GeneMark version 2.5 (Besemer \& Borodovsky, 2005) were used to predict open reading frames (ORFs). Gene locations and start sites were corrected using Phamerator and Starterator (Cresawn et al., 2011) during the manual annotation process.

\section{Functional Calls}

BLASTp (Altschul et al., 1990), using NCBI (Wheeler \& Bhagwat, 2007) and PhagesDB (Russell \& Hatfull, 2017) version 2.9.0 databases, was used to find functional relationships with either known bacteriophage proteins or all known proteins within Genbank, respectively, to determine gene function. Functional BLAST calls with sequence identities above 35\%, query coverage above $75 \%$, and E-values below $10^{-7}$ were prioritized as strong functional evidence. HHpred version 3.2.0 (Söding et al., 2005) was used to detect structure homology with known proteins in the Protein Data Bank (PDB). High sequence similarity was preferred, as well as high 
Characterization of phage Giantsbane

probability $(>80-90 \%)$, low E-values $\left(<10^{-3}\right)$, and high coverage $(>40-50 \%)$. CDD was used to find residue homology with known proteins presented in conserved domain models. TmHmm version 2.0 (Krogh et al., 2001) was used to predict the presence of transmembrane helices from the protein sequence. Only predictions with probabilities over 0.75 were considered.

\section{Batch ANI}

Batch ANI was conducted using the command line tool OAU (Lee et al., 2016) and the USEARCH algorithm (version 11). The resulting OrthoANI values were converted into a colored-heat map using Prism version 8 (Graphpad Software, CA, USA).

\section{SplitsTree}

SplitsTree (version 4.13.1) analysis was used to group cluster AU phages based on Pham similarity. Phage lineages were based off of an arbitrary point of origin and the distance between nodes reflects the relative genomic distance between the genomes.

\section{Repeated Sequence Discovery}

Repeated sequences were elucidated in Giantsbane using MEME Suite version 5.1.0 and Phamerator was used to locate the position of the sequences relative to genes. Phamerator was utilized again to observe conserved Phams and their order within genomes.

\section{RESULTS}

\section{A. globiformis-infecting phage found in soil}

Phage distribution depends on the host's natural habitat (Clokie et al., 2011); therefore soil was expected to contain phages that infect $A$. globiformis, a common soil bacterium. Numerous phage were successfully isolated on A. globiformis using direct isolation from bulk 
soil (Figure 1a). Spot tests confirmed that clearings were a result of phage lysis. One clearing was chosen for isolation and purification, and two consecutive plaque assays were performed to isolate phage Giantsbane (Figure 1b). The purified phage was amplified through MINI and MIDI lysate generation to create a concentrated phage stock (Table 1).

a.

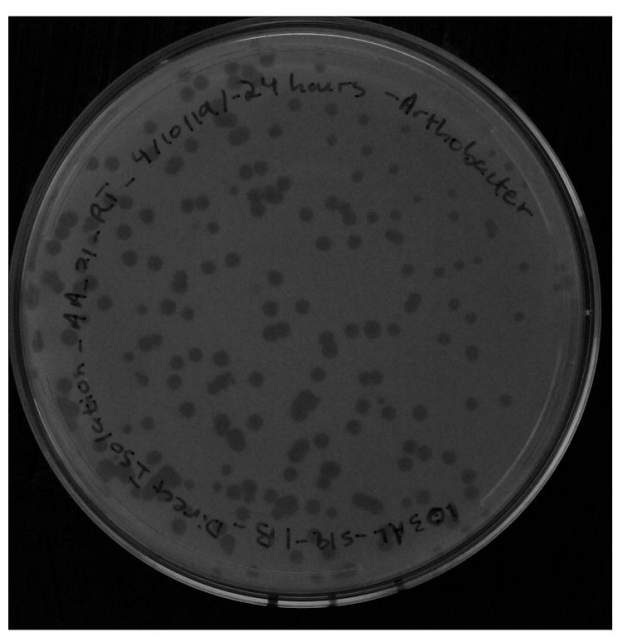

b.

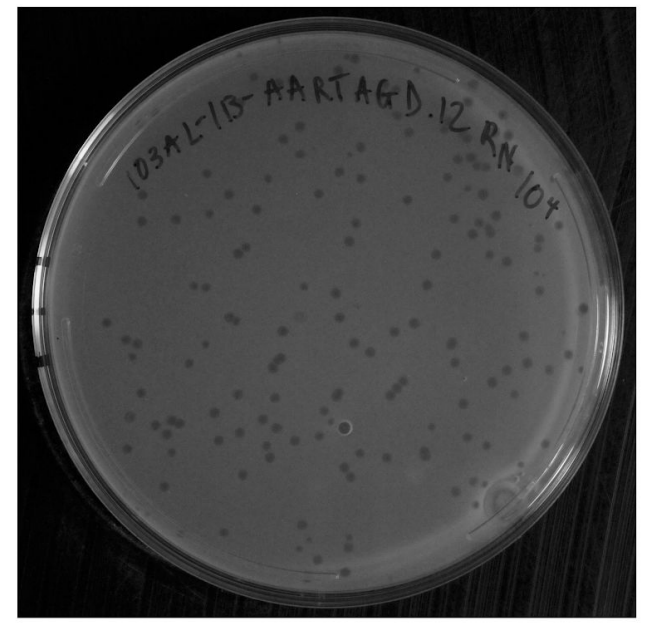

Figure $1 \mid \boldsymbol{A}$. globiformis-infecting phage plaque assays. a) Phage lysate isolated through direct isolation from the soil sample was plated on a soft agar overlay of PYCa soft agar and PYCa hard agar and incubated at $25^{\circ} \mathrm{C}$ for 24 hours. Putative plaques can be observed. b) A single clearing from the direct isolation plate was picked and purified through two plaque assays to isolate phage Giantbane. A representative plaque assay plate is shown. 
Characterization of phage Giantsbane

Table 1 | Results from amplification and purification of Giantsbane

\begin{tabular}{|c|c|c|c|}
\hline $\begin{array}{c}\text { Purification/ } \\
\text { Amplification } \\
\text { Iteration }\end{array}$ & $\begin{array}{c}\text { Avg. Titer } \\
\text { (PFU/mL) }\end{array}$ & Morphology & $\begin{array}{c}\text { Diameter } \\
\text { (mm) }\end{array}$ \\
\hline $1^{\text {st }}$ Purification & $1.5 \times 10^{7}$ & Bullseye & 1.0 \\
\hline $2^{\text {nd }}$ Purification & $4.2 \times 10^{7}$ & Bullseye & 1.0 \\
\hline MINI Lysate & $5.86 \times 10^{8}$ & Clear & 1.0 \\
\hline MIDI Lysate & $\begin{array}{c}4.9 \times 10^{7} \\
\pm 3.96 \times 10^{6}\end{array}$ & $\begin{array}{c}\text { Clear, } \\
\text { Bullseye }\end{array}$ \\
\hline
\end{tabular}

\section{TEM reveals Siphoviridae morphology}

Head and tail morphology are useful for classifying phages (Fokine \& Rossmann, 2014).

Since phage head and tail morphology are integral to overall morphological classification, if

Giantsbane is a member of Siphoviridae, then it should exhibit a long, non-contractile tail. TEM

showed that Giantsbane has a head diameter of $80.7 \pm 4.2 \mathrm{~nm}$ and a long, flexible tail of length

$219 \pm 15 \mathrm{~nm}$ with some kinked patterns (Figure 2). These measurements and morphology are consistent with the characteristics of T5-related Siphoviridae (Carstens, 2012). 


\section{Characterization of phage Giantsbane}

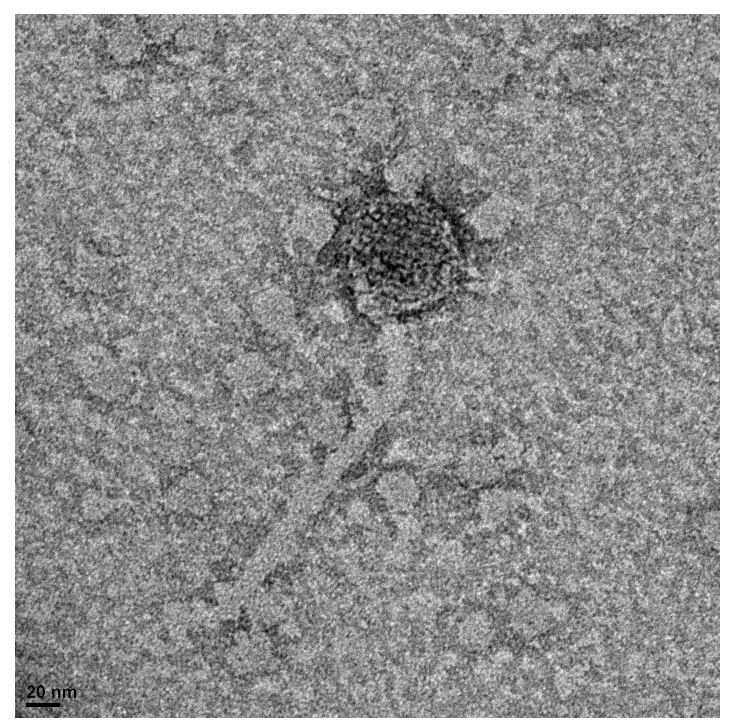

Figure 2 | Transmission electron microscope picture of Giantsbane reveals Siphoviridae morphology. A picture of Giantsbane's physical structure was taken using a Philips CM120 transmission electron microscope model at 52,000x magnification. The diameter of the phage head is approximately $80.7 \pm 4.2 \mathrm{~nm}$ and the length of the tail is approximately $219 \pm 15 \mathrm{~nm}$.

Phage infectivity is unaffected by $5^{\circ} \mathrm{C}$ deviation from $25^{\circ} \mathrm{C}$ and at salinities up to $0.12 \mathrm{M}$

The optimal growth temperature for $A$. globiformis is $25^{\circ} \mathrm{C}$ (Berger et al., 1996). To determine how conditions deviating from the host's preferred temperature affected phage propagation, plaque assay plates were incubated for 48 hours at various temperatures. Plaques appeared at $20^{\circ} \mathrm{C}, 25^{\circ} \mathrm{C}$, and $30^{\circ} \mathrm{C}$ (Figure 3). Titer was highest at $25^{\circ} \mathrm{C}$, but overall differences were statistically insignificant, indicating that small variations in temperature did not impact phage infectivity.

Soil salinity is highly variable on a temporal scale (Zhang et al., 2014), therefore, the effects of salinity concentration variations on phage propagation were also examined. Solutions of various $\mathrm{NaCl}$ concentrations were added to bacteria before a standard plaque assay was 
Characterization of phage Giantsbane

conducted. A negative correlation between titer and salinity was observed, but was not found to be statistically significant (Figure 4).

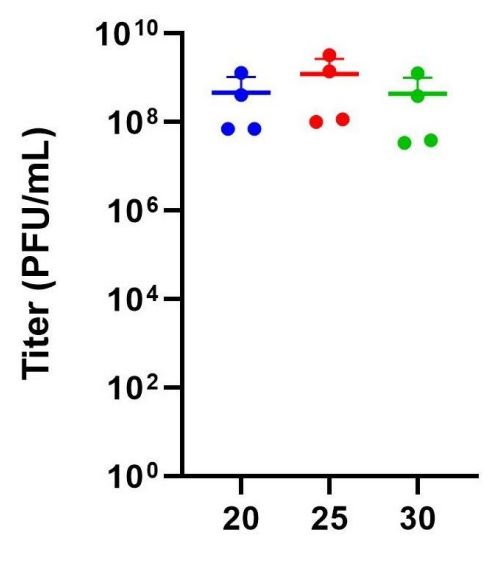

Temperature $\left({ }^{\circ} \mathrm{C}\right)$

Figure 3 | Incubation temperature does not affect titer. A. globiformis was inoculated with dilutions of phage lysate, plated, and incubated at $20^{\circ} \mathrm{C}, 25^{\circ} \mathrm{C}$ or $30^{\circ} \mathrm{C}$ for 48 hours. Plaque counts were used to calculate average titer. A decrease in titer can be observed in the $20^{\circ} \mathrm{C}$ and $30^{\circ} \mathrm{C}$ incubation temperatures compared to $25^{\circ} \mathrm{C}$, but was not found to be significant through a one-tailed unpaired Student's t-test at a 95\% confidence level. ( $\mathrm{n}=4$ trials)

\section{Giantsbane genome characteristics and AU2 classification}

Sequencing was performed on purified Giantsbane DNA using Illumina, with 2197x coverage. The genome was 56,734 bp in length, with a GC content of 50.1\% (Figure 5). The genome ends had 3' sticky overhangs, exhibiting a sequence of CGCCGGCCT. Giantsbane was categorized into cluster AU, which currently contains 20 phages, and subclustered with phage Shepard in AU2. Cluster AU phages are generally lytic, infect Arthrobacter and are classified as Siphoviridae, supporting Giantsbane's Siphoviridae classification as predicted by TEM. 
Characterization of phage Giantsbane

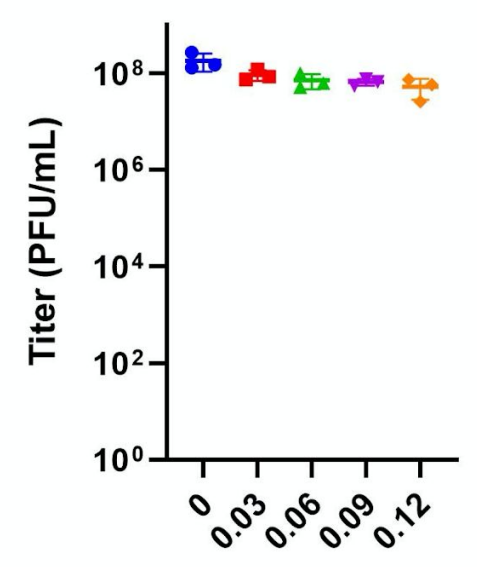

\section{Salinity Concentration ( $\mathrm{M} \mathrm{NaCl})$}

Figure 4 | Incubation of $\boldsymbol{A}$. globiformis and phage at varying salinities does not affect titer. A. globiformis was incubated in saline solutions at concentrations of $0,0.03,0.06,0.09$, and 0.12 $\mathrm{M} \mathrm{NaCl}$ before inoculation with phage. Plates were incubated at $25^{\circ} \mathrm{C}$ for 48 hours. Plaque counts were used to calculate average titer. A general decreasing trend in titer was observed as salinity concentration increases, but no variations in titer were found to be significant through a one-tailed unpaired Student's t-test at a 95\% confidence level. ( $\mathrm{n}=3$ trials)

\section{Manual correction of Giantsbane auto-annotation yields 94 putative genes}

After sequencing, auto-annotation and manual annotation were performed on the genome. Annotation resulted in 94 putative forward genes, 25 of which had putative functions, such as encoding for structural, packaging, and DNA replication proteins. The other 69 genes currently have no known functions (Figure 5). 


\section{Characterization of phage Giantsbane}

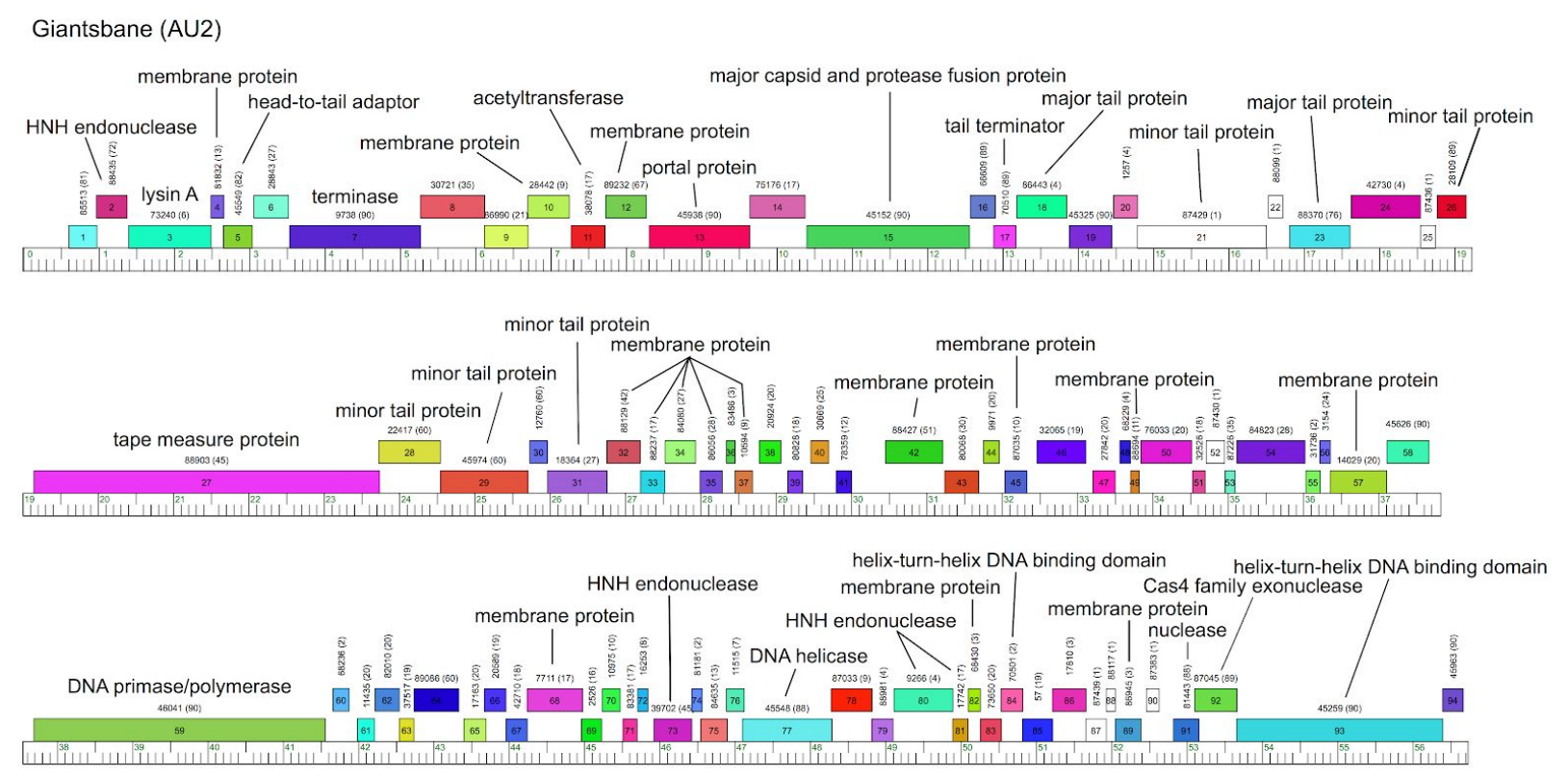

Figure 5 | Giantsbane Genome Map. The phage genome was auto-annotated using DNA Master and then manually corrected with additional genomic analysis programs. The finalized genome contains 94 putative ORFs, all in the forward direction. Notable genes include a Cas4 family exonuclease and a major capsid and protease fusion protein.

\section{Giantsbane shares highest nucleotide similarity with AU2 phage Shepard}

Phages are typically sorted into clusters and subclusters to show evolutionary relatedness and facilitate phage characterization. While phages are primarily clustered based on pham similarity, nucleotide similarity is an important factor as well (Hatfull, 2018). Batch ANI analysis with all AU phages helped examine intra-cluster similarities (Figure 6a). Giantsbane was found to share the highest percentage nucleotide similarity (86.6\%) with AU2 phage Shepard, and AU3 phages Ingrid and Loretta are also highly similar to each other. Unexpectedly, AU1 phage Makai shares higher nucleotide similarity with AU2 phages Giantsbane and Shepard (80.5\% and $82.2 \%)$ than with any other cluster AU phage, including its AU1 subcluster 
Characterization of phage Giantsbane

members. This suggests that subcluster AU1 has two distinct subgroups based on nucleotide similarity.

\section{SplitsTree results consistent with nucleotide sequence analysis}

Examining pham conservation between phages is the main factor for clustering phages.

Genes are grouped into phams based on amino acid sequence similarity, and phages sharing at least 35\% pham similarity are clustered together (Hatfull, 2018). Therefore, a SplitsTree analysis of pham conservation between Giantsbane and other AU phages was used to further examine intra-cluster similarities, which showed four distinct groups: the first containing Loretta and Ingrid; the second containing Makai, Giantsbane, and Shepard; the third containing Caterpillar and MediumFry, and a fourth containing all of the remaining AU1 phages (Figure 6b).

a.

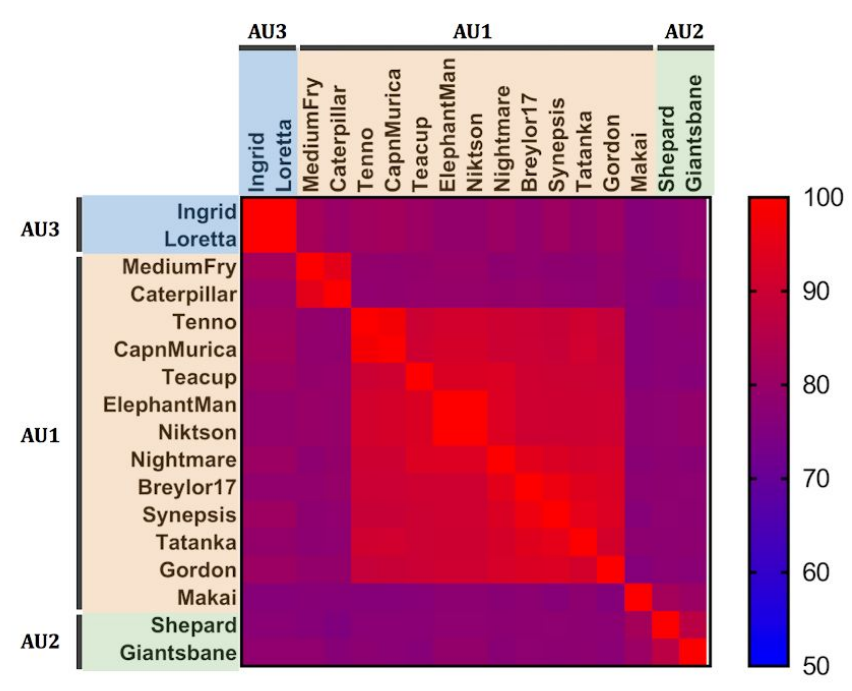

b.

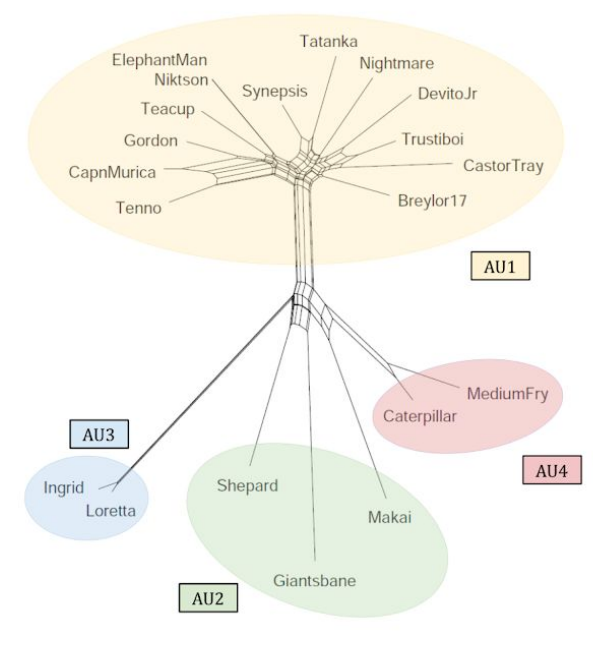

Figure 6 | Alternative groupings of AU phages based on nucleotide similarity and phams. a) A heat map was constructed based on the OrthoANI values obtained from Batch ANI analysis of cluster AU phages, with a color range of blue to red corresponding to nucleotide percentage similarities of $50 \%$ to $100 \%$, respectively. b) SplitsTree analysis was used to group cluster AU phages based on Pham similarity. The SplitsTree diagram showed four distinct groups within the AU cluster, as opposed to the three current subclusters. Makai was shown to be more closely 
Characterization of phage Giantsbane

related to both AU2 phages, compared to most AU1 phages, despite its classification in AU1, while AU1 Caterpillar and MediumFry are shown to be more closely related to each other than to any other AU1 phages.

\section{Giantsbane contains repeated sequences flanking conserved Phams of AU phages}

Nucleotide motifs, or repeat sequences, may be vestiges of recombination events (Rybnicky, 2017) and drive mosaicism in phages (De Paepe et al., 2014). As a result, looking into motifs in Giantsbane may elucidate recombination hotspots.

Using MEME to search for motifs within a single phage and Phamerator to look at conservation of motifs across genomes, a $32 \mathrm{bp}$ repeat sequence, indicated by red lines within Phamerator, was found in the intergenic regions surrounding genes 37-41 of Giantsbane (Figure 7a, 7b). This sequence occurs immediately upstream and downstream of each gene in this region, making it a boundary sequence as described by Clark et. al (2001); this pattern is found in AU1 and AU2 phages. The outer genes of this five-gene "cassette" were subject to translocation to different regions within the gene cassette (Figure 7c). This cassette contained the only instances of gene translocation observed among AU phage genomes. 


\section{Characterization of phage Giantsbane}

a.

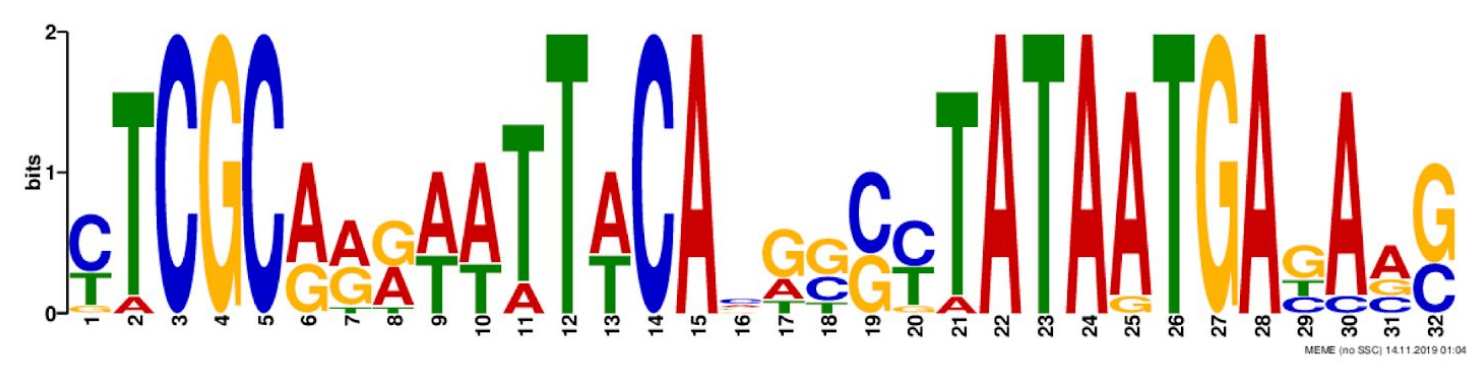

b.

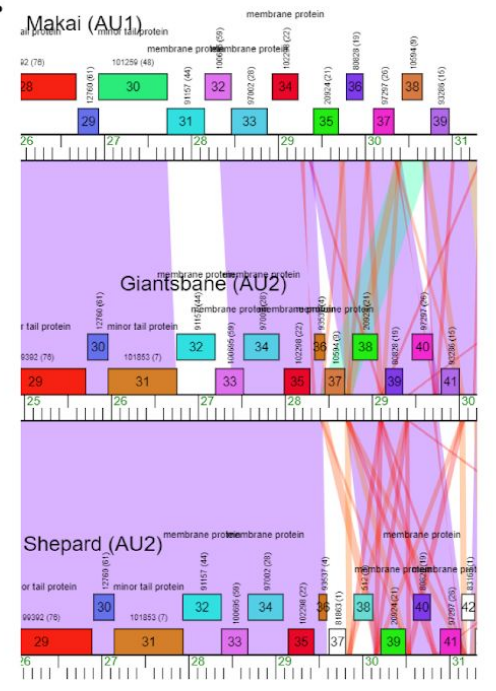

c.

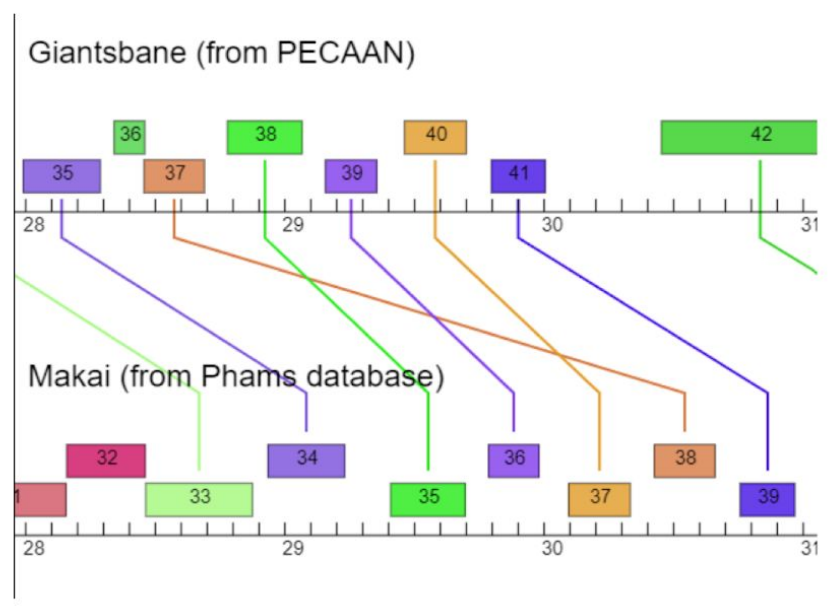

Figure 7 | Repeated sequence conserved among AU phages. Genomes of AU phages were analyzed using MEME and Phamerator to elucidate repeat sequences, their conservation across different phage genomes, and location relative to genes. a) A $32 \mathrm{bp}$ repeat was identified with MEME and displayed as a probability matrix that shows the likelihood of finding a nucleotide at that position in the sequence. b) Phamerator shows sequence similarity between multiple phage genomes. Purple indicates complete conservation and red lines show high conservation in certain regions. High degrees of conservation were found in the intergenic regions of the genome between 28.5-30 kbp. c) PECAAN output showing pham similarity between Giantsbane and Makai. Gene 37 of Giantsbane and gene 38 of Makai are part of the same pham but are in different locations relative to the other genes in this region, indicating translocation.

\section{DISCUSSION}

This research project provided a broad overview of the physical and genomic

characteristics of novel Actinobacteriophage Giantsbane. Phage biology is a relatively new field, 


\section{Characterization of phage Giantsbane}

requiring further characterization of novel bacteriophages. Our results add to pre-existing knowledge of Siphoviridae and Arthrobacter-infecting phages.

Environmental assays revealed that Giantsbane is unaffected by temperature and salinity variations based on titer analysis. Further research into environmental effects on Giantsbane would center on testing growth in different soil conditions, further elucidating its ability to survive in various conditions.

Genomic sequencing and annotation revealed 94 putative genes, including core structural, assembly, and lysis genes. No lysogenic genes were identified, supporting the classification of Giantsbane as a lytic phage. Currently, 69 genes have no known function. Batch ANI and SplitsTree analyses supported Giantsbane's placement into cluster AU. Giantsbane shares over $75 \%$ nucleotide similarity and high pham similarity with other AU phages. Cluster AU is composed of lytic Siphoviridae phages, suggesting that Giantsbane also exhibits these characteristics, which was confirmed with TEM analysis and the lack of a tail sheath protein.

Nucleotide similarity and pham conservation analyses revealed expected and unexpected patterns of inter-subcluster similarity. As expected, AU1 phages Caterpillar and MediumFry were observed to be more similar to each other than any other AU phage. However, AU1 phage Makai was found to have higher ANI with AU2 phages Shepard and Giantsbane than other AU1 phages. These unexpected inter-subcluster similarities allow for a better understanding of Arthrobacter-infecting Siphoviridae phages, which can help inform and clarify classification of similar phages in the future.

MEME and Phamerator revealed a $32 \mathrm{bp}$ repeat sequence in the intergenic regions of a five gene cluster in AU phages (excluding AU3) within the 28.5-30 kbp region. Genomic 
Characterization of phage Giantsbane

comparison analyses found that genes within this region were prone to insertions, deletions or translocations between phages. Several lambdoid phages have shown similar boundary sequences, proposed to create highly mosaic genomic regions (Clark et al., 2001). Gene translocations were only seen in this region, indicating the potential function of these intergenic repeat sequences. This provides evidence that boundary sequences may provide an additional means of recombination, in addition to illegitimate recombination (Morris et al., 2007). Multiple cycles of Giantsbane propagation followed by sequencing of the final generation and comparison with the original genome may help elucidate the potential importance of intergenic repeat sequences in mechanisms of phage genomic rearrangement and evolution. These findings would ultimately further our understanding of the roles of repeat sequences in phages and novel methods of propagating phage diversity.

\section{ACKNOWLEDGMENTS}

We thank Andy Alag for isolating Giantsbane; Krisanavane Reddi for preparation of materials and laboratory management; Yvette Lakkis and Andrew Lee for laboratory assistance; Hong Zhou of the UCLA Electron Imaging Center for NanoMachines for electron microscopy support; Rebecca A. Garlena and Daniel A. Russell at the Pittsburgh Bacteriophage Institute for genome sequencing and assembly; and Debbie Jacobs-Sera, Welkin Pope, Graham Hatfull, and the SEA-PHAGES community for programmatic support.

C.L., P.Y.C., P.D., and M.Z. drafted the paper and performed experiments; A.F. revised the paper; J.M.P. and A.F. supervised the research and performed quality control on the 
bioRxiv preprint doi: https://doi.org/10.1101/2020.01.10.891226; this version posted January 11, 2020. The copyright holder for this preprint

(which was not certified by peer review) is the author/funder, who has granted bioRxiv a license to display the preprint in perpetuity. It is made available under aCC-BY-NC-ND 4.0 International license.

\section{Characterization of phage Giantsbane}

annotations; and all authors contributed to the isolation, annotation, and genome analysis of the novel phages.

This project was funded by the Dean of Life Sciences Division at UCLA, with additional support for sequencing from the HHMI Science Education Alliance-Phage Hunters Advancing Genomics and Evolutionary Science (SEA-PHAGES) program. 


\section{Characterization of phage Giantsbane}

\section{REFERENCES}

Altschul SF, Gish W, Miller W, Myers EW, \& Lipman DJ. 1990. Basic local alignment search tool. Journal of Molecular Biology 215:403-410. doi:10.1016/S0022-2836(05)80360-2.

Berger F, Morellet N, Menu F, \& Potier P. 1996. Cold shock and cold acclimation proteins in the psychrotrophic bacterium Arthrobacter globiformis SI55. Journal of Bacteriology 178(11):2999-3007. doi:10.1128/jb.178.11.2999-3007.1996

Besemer J \& Borodovsky M. 2005. GeneMark: Web software for gene finding in prokaryotes, eukaryotes and viruses. Nucleic Acids Res 33:W451-W454. doi:10.1093/nar/gki487

Clark AJ, Inwood W, Cloutier T, \& Dhillon TS. 2001. Nucleotide sequence of coliphage HK620 and the evolution of lambdoid phages. Journal of Molecular Biology 311(4): 657-679. doi: 10.1006/jmbi.2001.4868

Carstens EB. 2012. Introduction to virus taxonomy. In King AMQ, Adams MJ, Carstens EB, \& Lefkowitz EJ. (Eds.), Virus Taxonomy (1-20). doi: 10.1016/B978-0-12-384684-6.00114-2.

Clokie MRJ, Millard AD, Letarov AV, \& Heaphy S. 2011. Phages in nature. Bacteriophage 1(1): 31-45. doi:10.4161/bact.1.1.14942

Cresawn SG, Bogel M, Day N, Jacobs-Sera D, Hendrix RW, \& Hatfull GF. 2011. Phamerator: a bioinformatic tool for comparative bacteriophage genomics. BMC Bioinformatics 12:395. doi:10.1186/1471-2105-12-395.

De Paepe M, Hutinet G, Son O, Amarir-Bouhram J, Schbath S, \& Petit MA. 2014. Temperate phages acquire DNA from defective prophages by relaxed homologous recombination: the role of Rad52-like recombinases. PLoS Genetics 10(3):e1004181. doi: 10.3389/fcimb.2014.00039

Delcher AL, Bratke KA, Powers EC, \& Salzberg SL. 2007. Identifying bacterial genes and endosymbiont DNA with Glimmer. Bioinformatics 23:673-679. doi:10.1093/bioinformatics/btm009

Eschbach M, Möbitz H, Rompf A, \& Jahn D. 2003. Members of the genus Arthrobacter grow anaerobically using nitrate ammonification and fermentative processes: Anaerobic 
Characterization of phage Giantsbane

adaptation of aerobic bacteria abundant in soil. FEMS Microbiology Letters 223:227-230. doi: 10.1016/S0378-1097(03)00383-5

Fokine A \& Rossmann MG. 2014. Molecular architecture of double-stranded DNA phages. Bacteriophage 4(1):e28281. doi: 10.4161/bact.28281

Hatfull GF. 2015. Dark Matter of the Biosphere: the Amazing World of Bacteriophage Diversity. Journal of Virology 89(16):8107-8110. doi: 10.1128/jvi.01340-15

Hatfull GF. 2018. Mycobacteriophages. Microbiology Spectrum, 6(5):1-32. doi:10.1128/microbiolspec.GPP3-0026-2018

Iriarte FB, Balogh B, Momol MT, Smith LM, Wilson M, \& Jones JB. 2007. Factors affecting survival of bacteriophage on tomato leaf surfaces. Applied and environmental microbiology 73(6):1704-1711. doi: 10.1128/AEM.02118-06

Jończyk E, Klak M, Międzybrodzki R, \& Górski A. 2011. The influence of external factors on bacteriophages-review. Folia Microbiologica 56(3):191-200. doi: $10.1007 / \mathrm{s} 12223-011-0039-8$

Krogh A, Larsson B, von Heijne G, \& Sonnhammer ELL. 2001. Predicting transmembrane protein topology with a hidden Markov model: application to complete genomes. J Mol Biol 305:567-580. doi:10.1006/jmbi.2000.4315.

Lee I, Kim YO, Park SC, \& Chun J. 2016. OrthoANI: An improved algorithm and software for calculating average nucleotide identity. International Journal of System and Evolutionary Microbiology 66(2):1100-1103. doi:10.1099/ijsem.0.000760

Morris P, Marinelli, LJ, Jacobs-Sera D, Hendrix RW, \& Hatfull GF. 2007. Genomic characterization of Mycobacteriophage Giles: Evidence for phage acquisition of host DNA by illegitimate recombination. Journal of Bacteriology 190(6):2172-2182. doi: 10.1128/JB.01657-07

Romaniuk K, Golec P, \& Dziewit L. 2018. Insight into the diversity and possible role of plasmids in the adaptation of psychrotolerant and metallotolerant Arthrobacter spp. to extreme Antarctic environments. Frontier Microbiology 9:3144. doi:

10.3389/fmicb.2018.03144 
Characterization of phage Giantsbane

Russell DA \& Hatfull GF. 2017. PhagesDB: the actinobacteriophage database. Bioinformatics 33(5):784-786. doi:10.1093/bioinformatics/btw711

Söding J, Biegert A, \& Lupas AN. 2005. The HHpred interactive server for protein homology detection and structure prediction. Nucleic Acids Res 33:W244-W248. doi:10.1093/nar/gki408.

Uroz S, Oger P, Lepleux C, Collignon C, Frey-Klett P, \& Turpault MP. 2011. Bacterial weathering and its contribution to nutrient cycling in temperate forest ecosystems. Research in Microbiology 162(9):820-831. doi: 10.1016/j.resmic.2011.01.013

Wheeler D \& Bhagwat M. 2007. BLAST QuickStart: example-driven web-based BLAST tutorial. Methods in Molecular Biology 395:149-176.

Woody MA \& Cliver DO. 1995. Effects of temperature and host cell growth phase on replication of F-specific RNA coliphage Q beta. Applied Environmental Microbiology 61(4):1520-1526. doi: 0099-2240/95/\$04.0010

Zhang J, Gao Q, Zhang Q, Wang T, Yue H, Wu L, Shi J, Qin Z, Zhou J, Zuo J \& Yang Y. 2017. Bacteriophage-prokaryote dynamics and interaction within anaerobic digestion processes across time and space. Microbiome 5(1). doi: 10.1186/s40168-017-0272-8

Zhang W, Wu H, Gu H, Feng G, Wang Z, \& Sheng J. 2014. Variability of soil salinity at multiple spatio-temporal scales and the related driving factors in the oasis areas of Xinjiang, China. Pedosphere 24(6):753-762. doi: 10.1016/S1002-0160(14)60062-X 